\title{
CONCENTRATION POLARIZATION PHENOMENA DURING DEAD-END ULTRAFILTRATION OF PROTEIN MIXTURES. THE INFLUENCE OF SOLUTE-SOLUTE INTERACTIONS
}

\author{
G.B. van den BERG* and C.A. SMOLDERS \\ Department of Chemical Technology, University of Twente, P.O. Box 217, 7500 AE Enschede \\ (The Netherlands)
}

(Received June 15, 1988; accepted in revised form February 10, 1989)

\begin{abstract}
Summary
The flux decline behaviour of some charged proteins and of binary mixtures of charged solutes during unstirred dead-end ultrafiltration has been studied. The mixtures consisted of the proteins bovine serum albumin, (BSA), $\alpha$-lactalbumin and/or lysozyme. Of special interest were $\alpha$-lactalbumin and lysozyme because these proteins are physico-chemically identical, except for the sign of their charge at the conditions used $\left(\mathrm{pH}=7.4, I=0.125 \mathrm{~N}\right.$ and $\left.T=20^{\circ} \mathrm{C}\right)$. 'I'he ultrafiltration properties were studied using the boundary layer resistance model. Ultrafiltration of single protein solutions of $\alpha$-lactalbumin and of lysozyme showed identical characteristics. The fouling behaviour during ultrafiltration of binary mixtures of the three components appeared to be dependent on both the charge of the solutes and the (unequal) dimensions of the solutes. A mixture of oppositely charged proteins (i.e., BSA/lysozyme or $\alpha$-lactalbumin/lysozyme) sometimes showed a considerable increase of the resistance of the concentrated layer near the membrane, depending on the mixing ratio of the two proteins. When equally charged (i.e., BSA $/ \alpha$-lactalbumin) proteins are ultrafiltered, a small decrease of the resistance could be observed, again depending on the mixing ratio of the proteins. The charge of the proteins, especially opposite charges, appeared to influence the flux behaviour more than the slightly denser packing of the solutes (as a result of unequal dimensions) would account for.
\end{abstract}

\section{Introduction}

Concentration polarization phenomena during membrane filtration have been described extensively in the last few decades. Several models have been proposed and these all appeared to be useful for the specific solutes under study. The models were often adequate for one class of solutes but could not explain the phenomena which occurred during filtration of other types of solutes. The appearance of a variety of other flux-lowering phenomena, such as adsorption, pore blocking and gel layer formation, may have been the reason for this shortcoming. The inappropriate background of a model could also be the origin of its failure, e.g., the use of specific characteristics of one solute may not be applicable to all solutes. The osmotic pressure model will not apply, for ex-

*Present address: Unilever Research Laboratory, Colworth House, Sharnbrook, Bedford MK44 1LQ, England. 
ample, in the case of filtration of colloidal suspensions. Sometimes the analysis of concentration polarization phenomena is done by more than one model at a time: e.g., Choe et al. [1] used the osmotic pressure model for dextrans and the classic (compressible) filter-cake model for the colloidal suspension of bentonite to distinguish the two types of filtration behaviour of dextrans and bentonite. On the other hand, several models have been used to describe the deadend ultrafiltration behaviour of one protein, i.e. bovine serum albumin (BSA): Vilker et al. [2] proposed the osmotic pressure model, Trettin and Doshi [3] the gel-polarization model, Reihanian et al. [4] and Chudacek and Fane [5] a particle filtration model and Van den Berg and Smolders [6] the boundary layer resistance model. The difference often is the number of assumptions concerning the concentration profile, the permeability of the concentrated layer and/or the presence of a gel layer. The nature of these models can vary from being more or less descriptive [4] to predictive [6].

Apart from the problem of choosing the best model for only one solute, there is the problem of describing the flux decline during the filtration of a complex fluid like milk or fruit juice. These liquids consist of many different solutes which can each have a different effect on flux decline. As shown by Ingham et al. [7] and Fane [8], the presence of large solutes can influence the retention of smaller solutes. Changing the ionic strength or the $\mathrm{pH}$ value of the solution, while using the same amount of macro-solute, can change the flux, which was also shown by Fane [8]. It will be clear that these changes can influence the properties of the solute and so the flux behaviour, indicating the importance of interactions between the micro- and macro-solutes in a solution. An example of macro-/macro-solute interaction can also be found: an increase in concentration of the protein $\beta$-lactoglobulin will result in an increasing retention because of association [9].

These examples clearly illustrate the problems which can arise when several solutes are brought together to simulate a complex fluid. The number of possible interactions that may occur during the filtration will increase exponentially with the increasing number of solutes. The total effect of the solutes on flux decline will thus be very difficult to predict quantitatively or even qualitatively.

The aim of this work is to show the effect of interactions between two different proteins during dead-end ultrafiltration. For that purpose, experimental filtration data will be analysed using the boundary layer resistance model as described by Nakao and co-workers $[10,11]$. The proteins studied were BSA, lysozyme and $\alpha$-lactalbumin. Of particular interest are the proteins lysozyme and $\alpha$-lactalbumin, because these two proteins are almost identical (in structure and geometry) but have totally different isoelectric points. The result is that, under the conditions used $(\mathrm{pH} 7.4)$, lysozyme is positive (net charge +7 groups per molecule) and $\alpha$-lactalbumin is negative (net charge -7). A mixture of these proteins, or a mixture of BSA (net charge -22) with one of these 
proteins, can therefore be of interest for a study of the interactions of proteins during dead-end ultrafiltration. Except for charge interactions, an additional effect can be expected to occur when mixtures are ultrafiltered: a different packing during solute build-up near the membrane interface. Therefore some model considerations for the packing of binary mixtures will be given.

\section{Theory}

\section{a. Data analysis by the Wijmans-Nakao model}

In their articles on the hydraulic boundary layer resistance model for ultrafiltration, Wijmans et al. [10] and Nakao et al. [11] showed that the analogy between permeation through a concentrated layer and sedimentation of a concentrated solution led to a model which could describe some concentration polarization phenomena near the membrane interface. Van den Berg and Smolders [6] adapted the boundary layer resistance model to the film theory and solved the differential equations involved numerically. The latter approach made it possible to predict the flux as a function of solute concentration, applied pressure, time and/or permeate volume, as well as predicting other flux decline-related phenomena such as the build-up of the concentrated layer near the membrane interface. The only experimental data needed were the resistance of the membrane for pure water flow and the independently determined diffusion and sedimentation coefficients of the solute as a function of concentration. However, when a mixture of solutes is used, the diffusion and sedimentation coefficients are hard to determine, and when also interactions occur it is impossible to calculate these coefficients. Johnston and Ogston [12] reported that, for a mixture of solutes in the absence of interaction, the sedimentation coefficient of each solute as a function of concentration is dependent on the total amount of solutes: when the mixture contains $x \mathrm{~g} / 1$ of solute $\mathrm{A}$ and $y \mathrm{~g} / \mathrm{l}$ of solute $\mathrm{B}$, the calculated sedimentation coefficients are $s_{\mathrm{A}}=s_{\mathrm{A}}(x+y)$ and $s_{\mathrm{B}}=s_{\mathrm{B}}(x+y)$, where $s_{\mathrm{A}}(C)$ and $s_{\mathrm{B}}(C)$ are normally different functions of concentration. When interactions occur, and more complex particles are formed, this kind of description is no longer valid. For this reason, the analysis of the experimental data will be done using the cake filtration approach [11].

The flux is given by:

$J_{\mathrm{v}}=\Delta P /\left[\eta_{\mathrm{o}}\left(R_{\mathrm{m}}+R_{\mathrm{bl}}\right)\right]$

where $\Delta P$ is the applied pressure, $\eta_{0}$ the viscosity of the solvent, $R_{\mathrm{m}}$ the resistance of the membrane and $R_{\mathrm{bl}}$ the resistance of the concentrated boundary layer.

The equivalent thickness of the boundary layer $\delta$, having a constant concentration $C_{\mathrm{bl}}$ in the cake filtration approach, can be obtained via the mass balance, resulting in: 
$C_{\mathrm{b}} \mathscr{R}_{\mathrm{obs}} V_{\mathrm{p}}=\delta A C_{\mathrm{bl}}$

in which $C_{\mathrm{b}}$ is the bulk concentration, $\mathscr{R}_{\mathrm{obs}}=1-\left(C_{\mathrm{p}} / C_{\mathrm{b}}\right)$ is the observed retention, $V_{\mathrm{p}}$ is the accumulated permeate volume and $A$ is the membrane area. Now the resistance of the boundary layer can be described by

$R_{\mathrm{bl}}=\delta r_{\mathrm{bl}}$

in which the specific resistance $r_{\mathrm{bl}}$ is assumed constant over the boundary layer $\delta$. Combining eqns. (1), (2) and (3) results in

$1 / J_{\mathrm{v}}=1 / J_{\mathrm{w}}+\left(\eta_{0} C_{\mathrm{b}} \mathscr{R}_{\mathrm{obs}} / \Delta P\right) \cdot\left(r_{\mathrm{bl}} / C_{\mathrm{bl}}\right) \cdot\left(V_{\mathrm{p}} / A\right)$

in which $\left(r_{\mathrm{bl}} / C_{\mathrm{bl}}\right)$ is a quantity called the flux decline index and $\left(V_{\mathrm{p}} / A\right)$ is the specific cumulative permeate volume.

In order to analyse the experimental results, for which slopes in the plot of $1 / J_{\mathrm{v}}$ as a function of $\left(V_{\mathrm{p}} / A\right)$ are required, eqn. (4) is transformed into:

$\mathrm{d}\left(1 / J_{\mathrm{v}}\right) / \mathrm{d}\left(V_{\mathrm{p}} / A\right)=\left(\eta_{0} C_{\mathrm{b}} \mathscr{R}_{\mathrm{obs}} / \Delta P\right) \cdot\left(r_{\mathrm{bl}} / C_{\mathrm{bl}}\right)$

For known values of $C_{\mathrm{b}}, \eta_{0}$ and $\Delta P$, the flux decline index $r_{\mathrm{bl}} / C_{\mathrm{bl}}$ can be determined from one set of experiments.

Eventually, from this $r_{\mathrm{bl}} / C_{\mathrm{bl}}$ value and the relationship between the permeability $p$ of the boundary layer and the sedimentation coefficient $s(C)$.

$p=1 / r_{\mathrm{bl}}=\left(\eta_{0} s(C) /\left[C\left(1-v_{1} / v_{0}\right)\right]\right.$

the boundary layer concentration $C_{\mathrm{bl}}$ can be calculated via

$r_{\mathrm{bl}} / C_{\mathrm{bl}}=\left(1-v_{1} / v_{0}\right) /\left[\eta_{0} s\left(C_{\mathrm{bl}}\right)\right]$

provided the dependence of $s$ on the concentration is known.

\section{b. Solute-solute interactions}

The interactions which can occur in a mixture of proteins in a buffer are both micro-/macro-solute interactions and macro-/macro-solute interactions. Since the same buffer (phosphate buffer at $\mathrm{pH} 7.4$ ) and the same amount of salt $(0.1 \mathrm{~N} \mathrm{NaCl})$ are used in all experiments in this work, the micro-/macrosolute interactions will be considered to be constant. The remaining (changing) interactions are the protein-protein interactions. These interactions can be subdivided into those that occur between proteins of one kind (self-association) and those that occur between different proteins.

When looking at the properties of the proteins used (Table 1) a number of characteristics can be observed. First of all, the striking resemblance of the proteins lysozyme and $\alpha$-lactalbumin is obvious; in this study, hen egg-white lysozyme and bovine $\alpha$-lactalbumin are used. These proteins have similar amino acid sequences, identical chain folds but different functions [21]. The physicochemical properties of these two proteins are therefore almost identical, except 
Physico-chemical properties of the proteins lysozyme, $\alpha$-lactalbumin and BSA (data are at $\mathrm{pH}=7.4$, $T=20^{\circ} \mathrm{C}$ and $I=0.125 \mathrm{~N}$, or closest data available)

\begin{tabular}{lccc}
\hline & Lysozyme & $\alpha$-Lactalbumin & BSA \\
\hline$M_{\mathrm{w}}$ (dalton) & $14,200[13]$ & $14,400[15]$ & $69,000[16]$ \\
& $14,100[14]$ & & \\
I.E.P. & $11.0[13]$ & $5.1[17]$ & $4.7[18]$ \\
Net charge at $\mathrm{pH}=7.4$ & $+7[13]$ & $-7[19]$ & $-22[20]$ \\
$D_{\mathrm{C}=0}\left(10^{-11} \mathrm{~m}^{2} / \mathrm{sec}\right)$ & $10.7[21]$ & $10.6[22]$ & $5.9[6]$ \\
$s_{\mathrm{C}=0}\left(10^{-13} \mathrm{sec}\right)$ & $1.86[21]$ & $1.83[17]$ & $4.41[6]$ \\
$v_{1}\left(10^{-4} \mathrm{~m}^{3} / \mathrm{kg}\right)$ & $7.26[23]$ & $7.35[22]$ & $7.34[18]$ \\
$r_{\mathrm{s}}(\mathrm{nm})$ & 2.00 & 2.02 & 3.64 \\
\hline
\end{tabular}

for the isoelectric point (I.E.P.) and therefore the net charge at $\mathrm{pH}$ 7.4. The consequence is that the filtration behaviour of the proteins separately has to be identical because the values of $s(C), D(C)$ and $v_{1}$ are the same, and those are the only parameters necessary to describe the dead-end filtration phenomena with the boundary layer resistance model.

In Table 1, the radius $r_{\mathrm{s}}$ of the proteins is calculated using the Stokes-Einstein equation for spherical particles

$D_{C=0}=k T /\left(6 \pi \eta r_{\mathrm{s}}\right)$

Self-association of the lysozyme molecules at high concentrations could introduce some problems when calculating the concentration in the boundary layer via eqn. (7), but since we use the measured sedimentation coefficient as a function of the actual concentration the "actual" boundary layer concentration will be calculated. The self-association constant for lysozyme is $K=0.489$ $\mathrm{m}^{3} / \mathrm{mol}$ at $\mathrm{pH} 7.0$ and $I=0.2 N[23]$.

Association between positively and negatively charged proteins can be expected to be substantially larger. Steiner et al. [24] found for the association constant of BSA and lysozyme at $\mathrm{pH} 7.0$ and $I=0.01 \mathrm{~N}$ a value of $40 \mathrm{~m}^{3} / \mathrm{mol}$. Although direct comparison between the association constants is not possible because of the difference in ionic strength, the association constant for the BSA-lysozyme couple seems to be considerably larger than for the self-association of the lysozyme proteins. No data were found in the literature concerning the association of $\alpha$-lactalbumin with other or identical proteins. The fact that mixtures of proteins carrying opposite charges form stable solutions indicates that not only these charge interactions are important, but also other factors, like for instance hydration of the protein molecules.

c. The build-up of a layer of particles of unequal size

Apart from the difference in charge, the solutes can also have different dimensions. When the stokes radius $(3.64 \mathrm{~nm}$ for BSA and $2.02 \mathrm{~nm}$ for $\alpha$-lac- 
talbumin and lysozyme) is taken as a reference, the diameter ratio is 0.55 when BSA is in the mixture and is 1.00 otherwise. From the literature, e.g., Ref. [25], it is known that mixing particles of different size will increase the overall packing density and will therefore enhance the resistance to permeation of solvent. The extent of this effect depends on the particle diameter ratio and the way of packing.

The packing density changes with the regularity of the lattices built up. For packing of equal spheres, the cubic-close-packed (c.c.p.) structure is the most dense, with only $25.9 \%$ porosity. In filtration a randomly packed layer of particles is more likely to be formed. Many experiments and computer simulations have been performed to calculate the porosity, $\epsilon$, of layers of spheres of equal size in random packing (see Rodriguez et al. [25] for a review). Depending on the coordination number, the packing density varies from $0.58(\epsilon=0.42)$ for "loose random packing" to $0.64(\epsilon=0.36)$ for "dense random packing" of equal spheres.

The packing of spheres of unequal size depends on the diameter ratio of the particles. For the case of a binary mixture, both experiments [26] and computer simulations $[25,27]$ have been performed for various diameter ratios. In general, the porosity or density is calculated as a function of the volume fraction $\phi_{1}$ of the smaller particles. Ben Aïm and Le Goff [26] showed experimentally that the porosity of a binary mixture of spheres with diameter ratio 0.52 (which is very close to our ratio of 0.55 for BSA and $\alpha$-lactalbumin or lysozyme) first decreases rapidly from $\epsilon=0.36$, at $\phi_{1}=0$, to a minimum value for the porosity $\epsilon=0.31$ at $\phi_{1}=0.25$, and increases slowly again to the original value of $\epsilon=0.36$ at $\phi_{1}=1$. A very similar behaviour is found in calculations by Dodds [27] for several diameter ratios (0.17 to 0.71$)$, although in this reference it was stated that "the absolute values of porosity were not realistic" $(\epsilon \approx 0.2)$. The calculations by Rodriguez et al. [25] of the packing density $(=1-\epsilon)$ as a function of $\phi_{1}$, at diameter ratio 0.3 , and comparison with several experimental values, showed rather large mutual deviations. The maximum density, at $\phi_{1} \approx 0.25$, increases from 0.64 (calculated [25,28]) to 0.74 (experimental [26]). Although this difference seems to be relatively small, when the Kozeny-Carman equation is used to calculate the specific resistance of such a layer the effect of the deviating density is very large. The Kozeny-Carman equation describes the specific resistance as [29]:

$r_{\mathrm{KC}}=\left[5\left(S_{0}\right)^{2}\right] \cdot\left[(1-\epsilon)^{2} / \epsilon^{3}\right]$

where $S_{0}$ is the specific area of the particles $\left(=3 / r_{\mathrm{s}}\right)$. For a given mixture with a fixed $\phi_{1}, S_{0}$ is constant, so that the specific resistance at $(1-\epsilon)=0.74$ is about 3.5 times larger than at $(1-\epsilon)=0.64$.

An estimation of the influence of the composition in a mixture of BSA (larger particles) and $\alpha$-lactalbumin or lysozyme (smaller particles) on the porosity and the specific resistance can be made using the experimental data for un- 
charged particles of Ben Aïm and Le Goff [26]. In this analysis we use the molar fraction as the main variable, as will be done in the plots in the section giving results of ultrafiltration experiments. In Fig. 1 the porosity and the specific resistance are given as a function of the molar fraction of small particles, assuming no interaction (a detailed description of mixtures with unequally sized, uncharged solutes is given in Appendix 1). The porosity clearly changes with the changing fraction of smaller particles $x_{1}$, with a minimum value near $x_{1}=0.6$, and the calculated specific resistance of a mixture of BSA and $\alpha$-lactalbumin or lysozyme seems to be an almost linear function of $x_{1}$. In this case (i.e., charge effects are excluded), the calculations for a mixture of $\alpha$-lactalbumin and lysozyme would result in a constant porosity $(\epsilon=0.36)$ and a constant specific resistance $\left(r \approx 1 \times 10^{20} \mathrm{~m}^{-2}\right)$.

An estimation concerning the behaviour of the flux decline index $r_{\mathrm{bl}} / C_{\mathrm{bl}}$ of a mixture of particles with the dimensions of BSA and $\alpha$-lactalbumin or lysozyme can also be made, while noticing that the computed values of the specific resistance are at least one order of magnitude too large (compare the values of the specific resistance given in figure A.1 with calculated values from Figs. 3 and 4). This can only be a very general estimation; the reasons are the unknown dependence of the quantity $r_{\mathrm{bl}} / C_{\mathrm{bl}}$ on the applied pressure and the unknown boundary layer concentration $C_{\mathrm{bl}}$. Assuming $C_{\mathrm{bl}}$ to be constant, which is true for $x_{1}=0$ and $x_{1}=1$ (see hereafter and Ref. [6]), the flux decline index is also a linear function of a molar fraction $x_{1}$. If the flux decline index of a

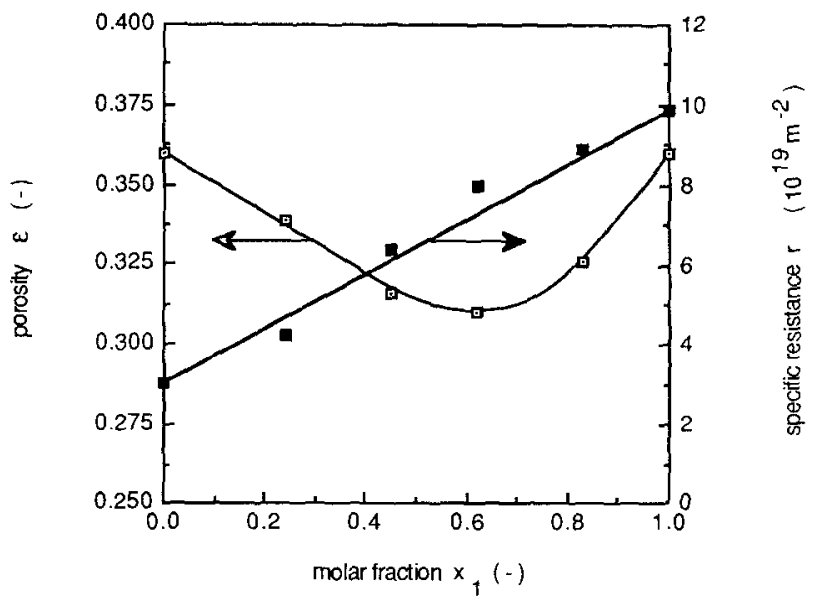

Fig. 1. Porosity and specific resistance (the latter according to the Kozeny-Carman equation) in a binary mixture of hard spheres as a function of the molar fraction of small particles. For the large particles the dimension of BSA $\left(d_{\mathrm{p}}=7.28 \mathrm{~nm}\right)$ is taken, and for the small particles the dimension of $\alpha$-lactalbumin or lysozyme $\left(d_{p}=4.0 \mathrm{~nm}\right)$ is taken. Interactions due to charge are considered to be absent. 
mixture of BSA and another protein as a function of the molar fraction of BSA follows a linear relationship we can write:

$r_{\mathrm{bl}} / C_{\mathrm{bl}}($ mixture of BSA + protein $x)$

$$
=x_{\mathrm{BSA}} r_{\mathrm{bl}} / C_{\mathrm{bl}}(\mathrm{BSA})+\left(1-x_{\mathrm{BSA}} r_{\mathrm{bl}} / C_{\mathrm{bl}}(\text { protein } x)\right.
$$

When the porosity decreases a little because of the unequal size of the solutes, e.g., as Ben Aïm and Le Goff described [26], the flux decline index will increase significantly, but as the concentration $C_{\mathrm{bl}}$ increases the flux decline index will also show a small decrease in comparison to eqn. (10). For the diameter ratio 0.52 the decrease in porosity and $r_{\mathrm{bl}} / C_{\mathrm{bl}}$ will be at most some $14 \%$.

\section{Experimental}

The proteins used were bovine serum albumin (BSA), hen egg-white lysozyme and bovine $\alpha$-lactalbumin. The BSA was a Cohn fraction V from Sigma Chemical Company. The crystallized lysozyme was from Fluka AG. The $\alpha$ lactalbumin was prepared from casein whey and was kindly supplied by the Netherlands Institute for Dairy Research (NIZO). The protein solutions were prepared in a phosphate buffer at $\mathrm{pH} 7.4 \pm 0.05$ with $0.1 \mathrm{M} \mathrm{NaCl}$ added, resulting in a solution with ionic strength $I=0.125 \mathrm{~N}$.

The concentration of each protein in the mixture was determined using a Waters HPLC system. The column used was a Protein-Pak 125 column (GPC), the detection wavelength was $280 \mathrm{~nm}$ and the buffer was a phosphate buffer of $\mathrm{pH} 7.4$ with $0.15 \mathrm{~N} \mathrm{Na}_{2} \mathrm{SO}_{4}$ added (for the separation of the equal molecular weight proteins $\alpha$-lactalbumin and lysozyme a buffer of $\mathrm{pH} 4.5$ was used).

The unstirred dead-end ultrafiltration experiments and the experiments to determine the sedimentation coefficient of lysozyme as a function of concentration were carried out as described previously [6]. The membranes used in the ultrafiltration experiments were Amicon YM-5 membranes (regenerated cellulose acetate, cut-off 5000 daltons). The retention for BSA was $100 \%$, and $99.5 \%+$ for the other proteins. No coagulation or flocculation was observed of non-concentrated solutions, in all possible mixtures, during ultrafiltration. This is also not very likely to occur when the association constants are considered (see part B of the theoretical section). The ionic strength of $0.125 \mathrm{~N}$ will prevent the proteins from coagulating at low concentrations at the $\mathrm{pH}$ used.

The concentration range for the determination of the sedimentation coefficients was 5 to $310 \mathrm{~kg} / \mathrm{m}^{3}$.

\section{Results and discussion}

\section{The sedimentalion coefficienl of lysozyme}

When the concentration in the layer near the membrane interface is calculated, the sedimentation coefficient as a function of concentration has to be 


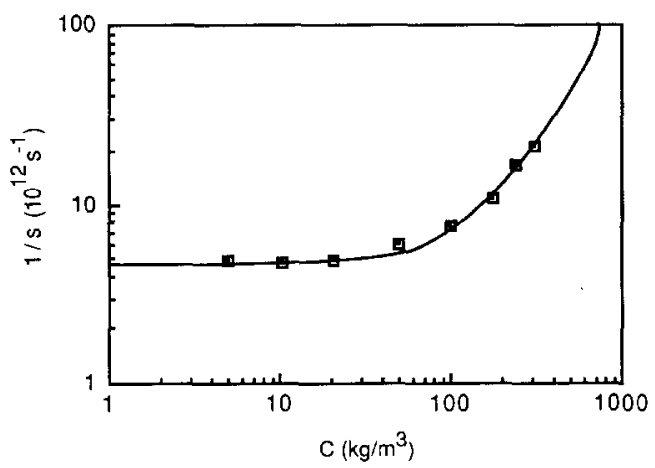

Fig. 2. The measured reciprocal sedimentation coefficient of lysozyme as a function of concentration $\left(\mathrm{pH}=7.4, I=0.125 \mathrm{~N}\right.$ and $\left.T=20^{\circ} \mathrm{C}\right)$.

known [eqn. (7)]. The sedimentation coefficient of lysozyme had to be determined experimentally because of the very scarce data on these coefficients in the literature, especially at higher concentrations. The values of the reciprocal sedimentation coefficient, as determined at $\mathrm{pH}=7.4, I=0.125 \mathrm{~N}$ and $T=20^{\circ} \mathrm{C}$, are represented in Fig. 2. Curve fitting of the experimental data resulted in:

$$
\begin{aligned}
1 / s=\left(1+1.067 \times 10^{-3} C+5.537 \times 10^{-5} C^{2}\right. & \\
- & \left.1.341 \times 10^{-7} C^{3}+1.856 \times 10^{-10} C^{4}\right) /\left(2.042 \times 10^{-13}\right)
\end{aligned}
$$

Following the strong similarity in physico-chemical properties of $\alpha$-lactalbumin and lysozyme, the sedimentation coefficient of $\alpha$-lactalbumin is assumed to have the same dependence on the concentration as applies for lysozyme. This dependence was not determined experimentally because of the limited amount of pure $\alpha$-lactalbumin available and its extraordinary high purchase price.

\section{Dead-end ultrafiltration of single protein solutions}

Ultrafiltration experiments with single protein solutions of lysozyme and $\alpha$ lactalbumin were performed to compare the filtration characteristics with those of BSA and with each other. The pressure used was 100 or $400 \mathrm{kPa}$, the temperature was $20^{\circ} \mathrm{C}$ and the concentration range was 0 to $4 \mathrm{~kg} / \mathrm{m}^{3}$ for $\alpha$-lactalbumin and 0 to $6 \mathrm{~kg} / \mathrm{m}^{3}$ for lysozyme.

As described in the theoretical section, the flux decline index $r_{\mathrm{bl}} / C_{\mathrm{bl}}$ can be calculated from the slope $\mathrm{d}\left(1 / J_{\mathrm{v}}\right) / \mathrm{d}\left(V_{\mathrm{p}} / A\right)$. In Fig. 3 the values of this index are given as a function of the concentration in the bulk for both the proteins lysozyme and $\alpha$-lactalbumin at the two applied pressures, 100 and $400 \mathrm{kPa}$.

From this figure it can be concluded that the flux decline indices are indeed equal for the two proteins, as could already be expected from the almost identical physico-chemical properties. As shown in our previous work for BSA [6] 


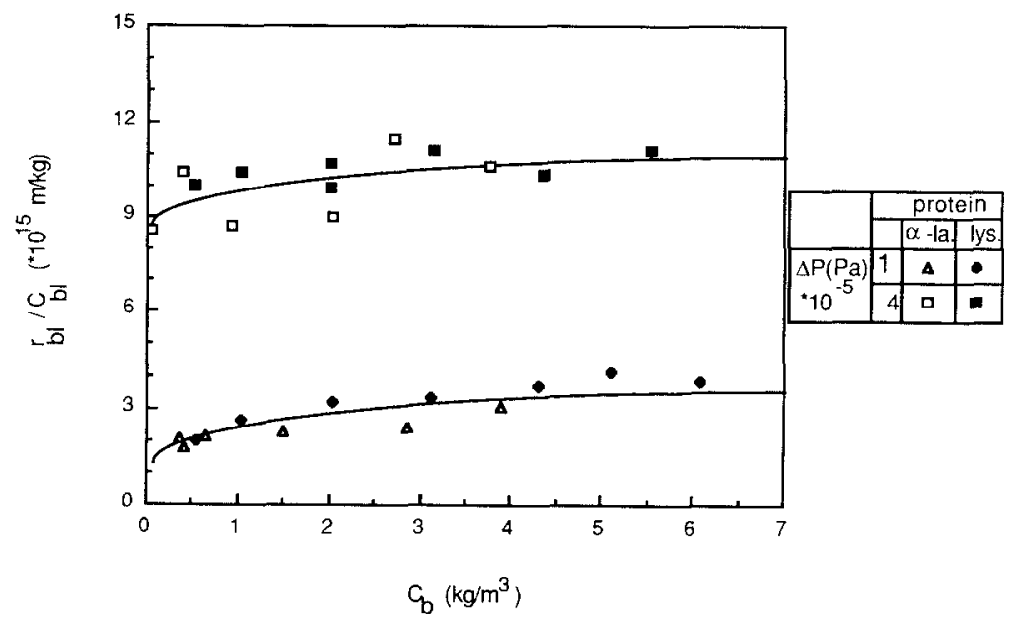

Fig. 3. The flux decline index $r_{\mathrm{bl}} / C_{\mathrm{bl}}$ as a function of concentration in the bulk for the proteins $\alpha$ lactalbumin and lysozyme. $\Delta P=100$ or $400 \mathrm{kPa}$.

the flux decline index also first increases with concentration at low concentration in the bulk but soon reaches a plateau value. These plateau values are about $3.9 \times 10^{15}$ and $10.8 \times 10^{15} \mathrm{~m} / \mathrm{kg}$, respectively, for the applied pressures 100 and $400 \mathrm{kPa}$. For BSA, values of $3.8 \times 10^{15}$ and $10.5 \times 10^{15} \mathrm{~m} / \mathrm{kg}$, respectively, were found at the pressures mentioned [6], As the values do not differ very much for these three proteins, the question arises whether the flux decline index could perhaps be about the same for all solutes at a given pressure. In Table 2 a number of flux decline indices is given for different kinds of solutes at applied pressures $\triangle P=100$ and $400 \mathrm{kPa}$ (most data were obtained from data points in plots, which makes them approximate values ).

Dejmek [30] found almost identical values for the specific "gel" resistance $(m)$ of the proteins haemoglobin and $\beta$-lactoglobulin $\left[r_{\mathrm{bl}} / C_{\mathrm{bl}}=(m) / \eta_{0}\right]$. Nakao et al. [11] calculated much higher values for the flux decline index of the solutes Dextran T500 and PEG 600. On the other hand, data reported by McDonogh et al. [31] for silica colloids with varying zeta potentials show much lower values.

The almost identical values for the proteins and the different values for the other solutes seem to lead to the conclusion that the permeability of a concentrated boundary layer depends on the compactness and type of packing of these solutes. A concentrated layer of the rather compact and impermeable protein molecules is more permeable than a layer of entangled polymers (Dextrans and PEG), but less permeable than a layer of very compact but rather loosely packed silica colloids. The observation by McDonogh et al. that a higher zeta potential (more open structure) decreases the specific resistance of colloids agrees with this conclusion. In the case of Dextran T70 and PEG 600 (above the overlap concentration, which is about 5\%) the transport of the solvent 
TABLE 2

The flux density index of several solutes at applied pressures $\Delta P=100$ and $400 \mathrm{kPa}$; most data taken from the literature

\begin{tabular}{llrl}
\hline Solute & $\Delta P\left(10^{5} \mathrm{~Pa}\right)$ & $\frac{r_{\mathrm{bl}}}{C_{\mathrm{bl}}}\left(10^{15} \mathrm{~m} / \mathrm{kg}\right)$ & Reference \\
\hline Lysozyme or & 1 & 3.9 & \\
$\quad \alpha$-lactalbumin & 4 & 10.8 & this work \\
BSA & 1 & 3.8 & {$[6]$} \\
& 4 & 10.5 & \\
Haemoglobin & 1 & 3.8 & {$[30]$} \\
$\beta$-Lactoglobulin & 4 & 11.5 & {$[30]$} \\
Dextran T500 & 1 & 3.1 & {$[11]$} \\
PEG 600 & 4 & 9.4 & {$[11]$} \\
Silica colloids & 1 & 13.5 & {$[31]$} \\
\hline
\end{tabular}

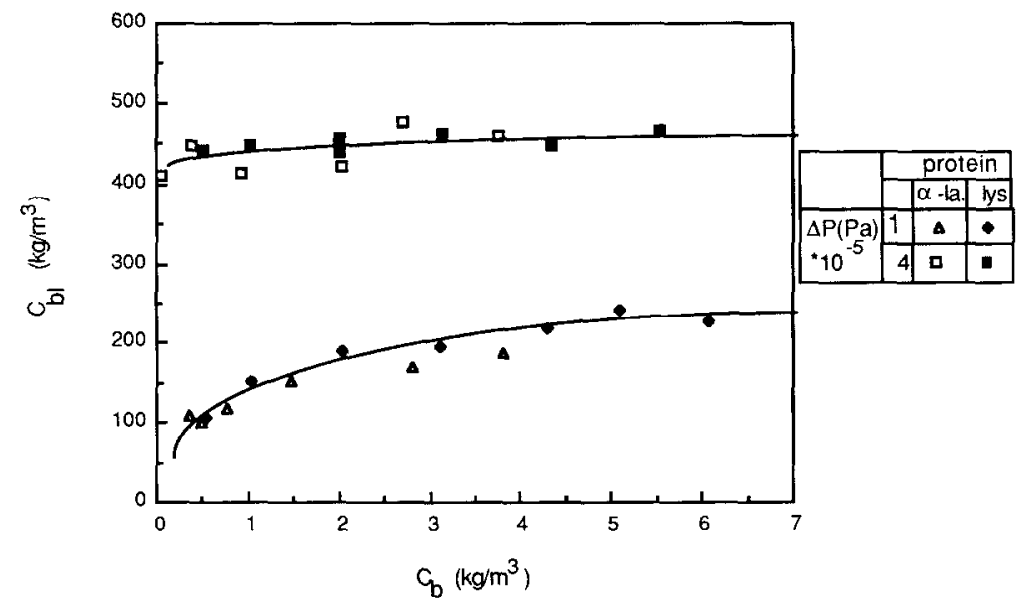

Fig. 4. The calculated boundary layer concentration $C_{\mathrm{bl}}$ as a function of the initial bulk concentration and the applied pressure.

water probably occurs through the molecular coils (intramolecular), while the transport is around the particles (intermolecular) in the case of proteins and colloids [32].

When the data from Fig. 3 are used to calculate the concentration in the boundary layer, with the help of eqns. (7) and (11), it can be seen that these concentrations can reach quite high values (Fig. 4). In these calculations eqn. (11) is used both for lysozyme and for $\alpha$-lactalbumin. The plateau values of 
the boundary layer concentrations are about 240 and $450 \mathrm{~kg} / \mathrm{m}^{3}$ for the applied pressures $\Delta P=100$ and $400 \mathrm{kPa}$, respectively. It will be clear that at these high concentrations quite large resistances to permeation can be expected. The real value of the concentration at the membrane interface will be different from those mentioned here because of the assumption that the concentration in the boundary layer is constant, i.e. independent of the distance. In our previous work [6] we showed that a decreasing concentration profile into the bulk solution is more realistic, and can be calculated from basic equations for the case of a solution with one solute. In that case, the concentration at the membrane interface was shown to be somewhat higher. There is a maximum possible concentration, of course, which is determined by the density of the particles, the shape and size of the particles and the interparticle distance as a result of attraction or repulsion.

\section{Dead-end ultrafiltration of mixtures of proteins}

'The flux decline indices of mixtures of proteins are calculated in the same way as for solutions of single proteins. To make allowance for the specific properties of each kind of protein (e.g., charge and molecular weight) the flux decline index is represented as a function of the number of moles present in the solutions: the molar fraction of one of the proteins is used as composition variable.

As mentioned in the theoretical section, the flux decline index of a mixture of BSA and another protein as a function of the molar fraction of BSA possibly follows a linear relationship, given by eqn. (10). Obviously, if the experimental data followed this linear relationship, there would be no difference between the interaction of a protein with a protein of the same kind and the interaction with a differently charged and differently sized protein (e.g., diameter ratio 0.55 for the proteins used).

In Fig. 5 the indices are given as a function of the molar fraction of BSA present in mixtures with either lysozyme or $\alpha$-lactalbumin. The data at $x=0$ and $x=1$ are the plateau values for the flux decline indices of the single proteins.

For the two pressures studied, the values for the flux decline index of a mixture of BSA and $\alpha$-lactalbumin (both negatively charged) show slightly smaller values than the linear curve representing eqn. (10). In view of the theoretical considerations given above, this would mean that the BSA and $\alpha$-lactalbumin molecules have built up a concentrated layer during filtration, with a slightly decreased flux decline index in comparison with two non-interacting proteins in the mixture. The repulsion between these different molecules is almost equal to the repulsion between two proteins of only one kind. The change to a more loosely packed boundary layer could perhaps be due to the non-spherical dimensions of the BSA molecule. Even so, our conclusion is that essentially the experimentally determined flux decline indices show the same tendency here with changing molar fraction $x_{1}$ as derived in the theoretical section, indicating 


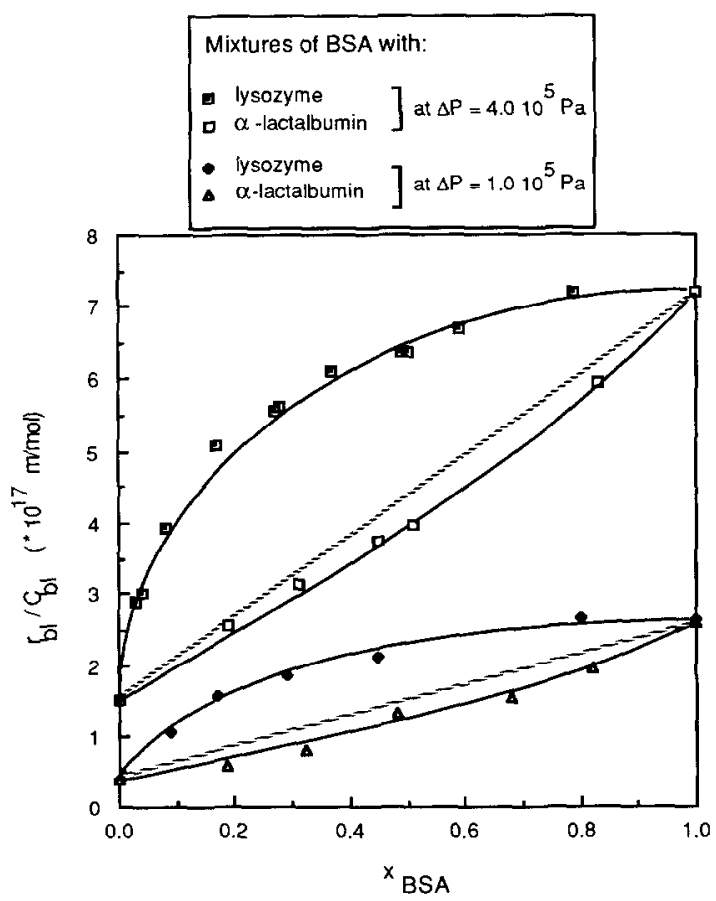

Fig. 5. The flux decline index $r_{\mathrm{bl}} / C_{\mathrm{bl}}$ (on a molar basis) for mixtures of BSA with $\alpha$-lactalbumin or lysozyme, as a function of the molar fraction of BSA in the solution. $\Delta P=100$ and $400 \mathrm{kPa}$, $T=20^{\circ} \mathrm{C}, \mathrm{pH}=7.4$ and $I=0.125 \mathrm{~N}$. The concentration in the bulk solution was between 1 and 5 $\mathrm{kg} / \mathrm{m}^{3}$.

that the equations originally meant for much larger spherical particles can also be applied to hard semispherical solutes such as proteins.

The data on the mixtures of BSA and lysozyme show a quite different flux behaviour. The flux decline index is larger than that given by a linear relationship between the indices of the single protein solutions [eqn. (10). The index can reach values up to twice the predicted value (i.e. for $x_{\mathrm{BSA}} \approx 0.1$ to 0.2 ). The origin of the larger resistance than that predicted for non-interacting particles may be found in a tighter packing of the concentrated layer. This is possible because of the oppositely charged particles. It can be envisaged that a boundary layer of positively and negatively charged proteins can be packed more densely than a layer of protein molecules with just one type of charge, resulting in a much higher specific resistance. While the flux decline index $r_{\mathrm{bl}} / C_{\mathrm{bl}}$ increases more than linearly with $x_{\mathrm{BSA}}$, the specific resistance $r_{\mathrm{b}}$ will have to increase still more markedly, since the concentration $C_{\mathrm{bl}}$ can also be expected to increase as a result of the attraction. These observations can also be described theoretically for the analogous case of single lysozyme solutions, using the equations for calculation of the specific resistance [eqns. (7) and (11)]. When 
the concentration in the boundary layer increases, e.g., from 300 to $360 \mathrm{~kg} / \mathrm{m}^{3}$ ( $20 \%$ increase), the specific resistance will increase more strongly because of the exponential equation for the (reciprocal) sedimentation coefficient (in this example $30 \%$ ) resulting in a higher value for the flux decline index (in this example $8 \%$ ).

In Fig. 6 the results for the experiments using mixtures of the equally sized but oppositely charged $\alpha$-lactalbumin and lysozyme are shown. For these mixtures the flux decline index again is considerably larger than the values for the single protein solutions and seems to indicate a maximum near $x_{\alpha \text {-lactalbumin }}=0.5$. Here the flux decline index appears to be about 2.3 times as large as in the case of single protein solutions. The packing of the positively and negatively charged protein molecules will again be much more dense than in the case of the single protein solutions. Except for the sign of the charge, these molecules are totally identical as to physico-chemical properties and flux behaviour (Table 1 and Fig. 3). Hence the specific resistance or the flux decline index could be expected to be totally constant if the attraction were not present. The effect of oppositely charged but otherwise identical molecules in a network can only be an enhanced packing density and an increasing resistance, probably with a maximum in resistance at $x_{\alpha \text {-lactalbumin }}=0.5$ because of the numerically equal charge for lysozyme and $\alpha$-lactalbumin and identical dimensions for these proteins. Looking only at mutual charge compensation for an

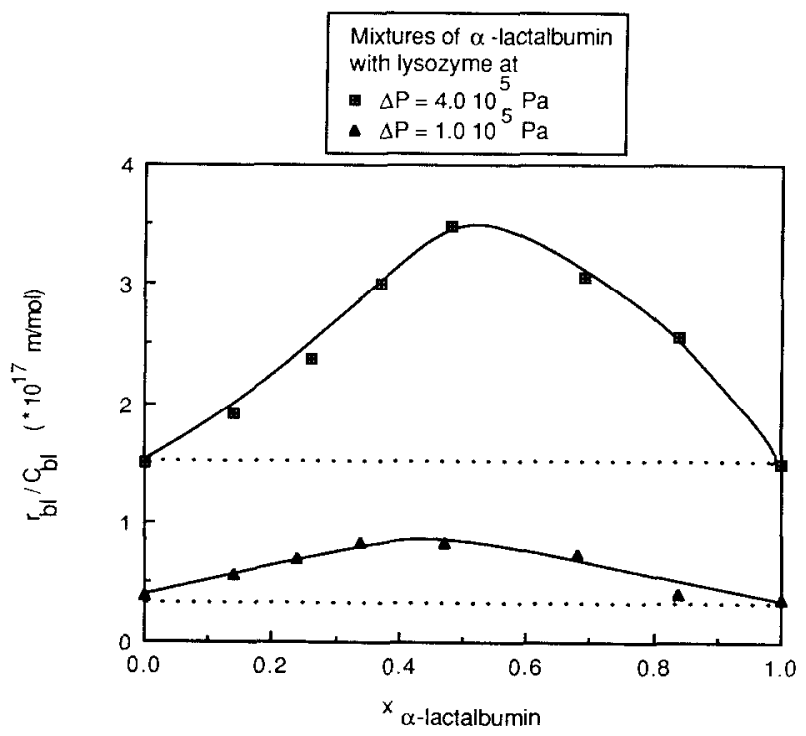

Fig. 6. The flux decline index $r_{\mathrm{bl}} / C_{\mathrm{bl}}$ (on a molar basis) for mixtures of $\alpha$-lactalbumin with lysozyme, as a function of the molar fraction of $\alpha$-lactalbumin in the solution. $\Delta P=100$ and $400 \mathrm{kPa}$, $\mathrm{pH}-7.4, T=20^{\circ} \mathrm{C}$ and $I=0.125 \mathrm{~N}$ 
effect on packing density (and not at the influence of particle dimensions), the maximum relative increase in resistance for the case of mixing lysozyme and BSA could be expected at $x_{\mathrm{BSA}}=0.25$. This is not too far from the actual situation found in Fig. 5.

The observation that the flux decline indices and specific resistances of mixture with oppositely charged particles only increase indicates that no coagulation or flocculation occurs in the solution before the particles settle in the concentrated layer near the membrane interface. If this had occurred the structure of the concentration polarization layer would have been much more open, with a lower resistance as the result [31]. The probable reason for this phenomenon is the presence of stabilizing hydration shells around the proteins.

The interparticle distance can be estimated for both the single protein solutions and the mixtures (see Appendix 2). The distance between molecules in a single protein solution is calculated to be $0.92 \mathrm{~nm}$, whereas the minimum distance in a mixture of $\alpha$-lactalbumin and lysozyme, at $x_{\alpha \text {-lactalbumin }}=0.5$, is only $0.44 \mathrm{~nm}$. Apparently a considerable decrease can occur due to the attraction of the oppositely charged proteins.

When the charge of the proteins changes, e.g., as a result of a change in $\mathrm{pH}$, different behaviour can be expected: when the charge of the positive lysozyme proteins is twice that of the negative $\alpha$-lactalbumin proteins, the maximum resistance can be expected at $x_{\alpha \text {-lactalbumin }}=0.67$ [since overall electroneutrality will exist only when the charge of one lysozyme molecule is counterbalanced by that of two $\alpha$-lactalbumin molecules, then $x_{\alpha \text {-lactalbumin }}=2 /(2+1)=0.67$ ] Although this example can be calculated easily, much more complicated calculations can be expected when the size of the solutes is unequal or when (many) more solutes are present in the solution. Then the total fouling capacity has to be determined using all the different interactions between the solutes, and their different sizes have to be taken into account as well, which probably results in too complex a set of equations. The only way then available is the experimental method to determine the flux decline index, which will also be of more use when other practical circumstances (e.g., the temperature) are changed.

\section{Conclusions}

The flux behaviour of mixtures of proteins during unstirred dead-end ultrafiltration can be very different from the behaviour of the single proteins. Both larger and about equal flux decline indices can be determined for solutions with mixtures of solutes, compared with the single solute solutions. The net charge of the molecules can give essential information on the kind of interactions which will lead to a different fouling behaviour. When oppositely charged proteins (lysozyme with $\alpha$-lactalbumin or BSA) are present in a mixture during ultrafiltration, the permeability of the concentrated boundary layer near the 
membrane interface can decrease drastically; this depends on the mixing ratio of the proteins. When equally charged proteins are present in a solution (BSA with $\alpha$-lactalbumin ) the resistance or flux decline index decreases only slightly. The effect of opposite charges seems to be much more effective than the difference in size of the solute molecules. The changing packing of layers of unequally sized particles certainly influences the resistance of such a layer, but not as much as the difference in charge does. Simulating the ultrafiltration of a complex liquid, like milk or whey, which has many differently sized and charged molecules, will therefore be very difficult. From the observations described above it will be clear that the fouling capacity of a mixture of solutes cannot be described by simply adding the fouling behaviour of the single solutes. When the total fouling capacity of a complex mixture has to be determined, the calculations will probably be too complex because of all the interactions between the solutes and the different sizes which have to be taken into account. The only way left then is the experimental approach, which will also be of more use when other practical circumstances, such as a changing $\mathrm{pH}$ value, are considered.

\section{Acknowledgements}

The authors wish to thank the Netherlands Institute for Dairy Research for supplying the $\alpha$-lactalbumin sample. Thanks also to Ms. A.Gonlag and Ms. M. Veldhuis for performing the experiments and analyses and to Dr. I.G. Rácz and Dr. Th. van den Boomgaard for helpful discussions.

\section{List of symbols}

$\begin{array}{ll}A & \text { membrane area }\left(\mathrm{m}^{2}\right) \\ C_{\mathrm{b}} & \text { concentration in the bulk }\left(\mathrm{kg} / \mathrm{m}^{3}\right) \\ C_{\mathrm{bl}} & \text { (constant) concentration in the boundary layer }\left(\mathrm{kg} / \mathrm{m}^{3}\right) \\ C_{\mathrm{p}} & \text { concentration in the permeate }\left(\mathrm{kg} / \mathrm{m}^{3}\right) \\ d_{\mathrm{p}} & \text { particle diameter }(\mathrm{m}) \\ D & \text { diffusion coefficient }\left(\mathrm{m}^{2} / \mathrm{sec}\right) \\ I & \text { ionic strength }(N) \\ \text { I.E.P. } & \text { isoelectric point }(-) \\ J_{\mathrm{v}} & \text { flux }\left(\mathrm{m}^{3} / \mathrm{m}^{2}-\mathrm{sec}\right) \\ J_{\mathrm{w}} & \text { clean water flux }\left(\mathrm{m}^{3} / \mathrm{m}^{2}-\mathrm{sec}\right) \\ m_{1,2} & \text { mass of smaller and larger particles, respectively }(\mathrm{kg}) \\ (m) & \text { specific gel resistance as defined by Dejmek }[30]\left(\mathrm{sec}^{-1}\right) \\ p & \text { permeability of the boundary layer }\left(\mathrm{m}^{2}\right) \\ r_{\mathrm{bl}} & \text { specific resistance of the boundary layer }\left(\mathrm{m}^{-2}\right) \\ r_{\mathrm{H}} & \text { specific resistance according to Happel's model }\left(\mathrm{m}^{-2}\right)\end{array}$




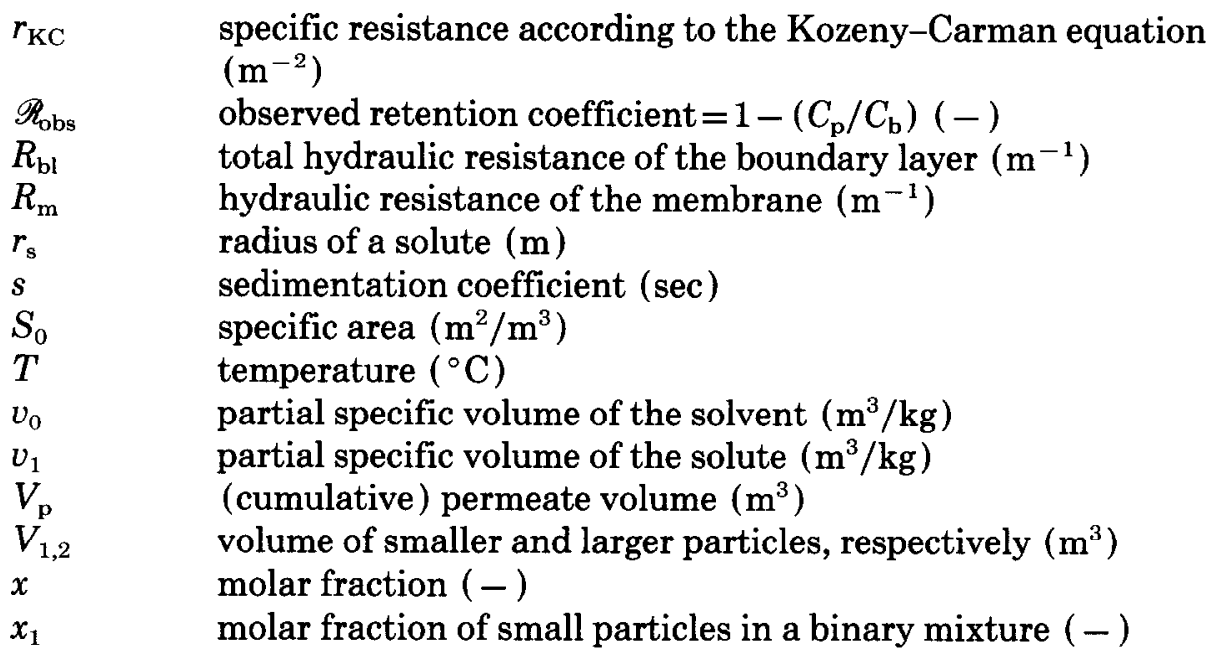

Greek letters

$\delta$

$\epsilon$

$\epsilon_{\text {app }}$

$\epsilon_{\mathrm{m}}$

$\epsilon_{\text {tot }}$

$\phi_{1}$

$\varphi_{\mathrm{e}}$

$\Delta P$

$\eta_{0}$ thickness of the boundary layer $(\mathrm{m})$

porosity (-)

apparent porosity (calculated from experimental data) (-) porosity of a mixture (-) total porosity of a layer of randomly packed partly filled spheres $(-)$

volume fraction of the small particles in a binary mixture $(-)$ term used in eqns. (A4) and (A5) (-)

applied pressure $(\mathrm{Pa})$

viscosity of the solvent $(\mathrm{Pa} \mathrm{sec})$

\section{References}

1 T.B. Choe, P. Masse, A. Verdier and M.J. Clifton, Flux decline in batch ultrafiltration: concentration polarization and cake formation, J. Membrane Sci., 26 (1986) 1-15.

2 V.L. Vilker, C.K. Colton and K.A. Smith, Concentration polarization in protein ultrafiltration. Part II. 'Theoretical and experimental study of albumin ultrafiltered in an unstirred batch cell, AIChE J., 27 (1981) 637-645.

3 D.R. Trettin and M.R. Doshi, Ultrafiltration in an unstirred batch cell, Ind. Eng. Chem. Fundam., 19 (1980) 189-194.

4 H. Reihanian, C.R. Robertson and A.S. Michaels, Mechanisms of polarization and fouling of ultrafiltration membranes by proteins, J. Membrane Sci., 16 (1983) 237-258.

5 M.W. Chudacek and A.G. Fane, The dynamics of polarisation in unstirred and stirred ultrafiltration, J. Membrane Sci., 21 (1984) 145-160.

6 G.B. van den Berg and C.A. Smolders, The boundary layer resistance model for unstirred ultrafiltration. A new approach, J. Membrane Sci., 40 (1989) 149-172. 
7 K.C. Ingham, T.F. Busby, Y. Sahlestrom and F. Castino, Separation of macromolecules by ultrafiltration: influence of protein adsorption, protein-protein interactions and concentration polarization, in: A.R. Cooper (Ed.), Ultrafiltration membranes and applications, Plenum Press, New York, NY, 1980, pp. 141-158.

8 A.G. Fane, Factor affecting flux and rejection in ultrafiltration, J. Sep. Process Technol., 4 (1983) 15-23.

9 G.B. van den Berg, J.H. Hanemaaijer and C.A. Smolders, Ultrafiltration of protein solutions; the role of protein association in rejection and osmotic pressure, J. Membrane Sci., 31 (1987) $307-320$.

10 J.G. Wijmans, S. Nakao, J.W.A. van den Berg, F.R. Troelstra and C.A. Smolders, Hydrodynamic resistance of concentration polarization boundary layers in ultrafiltration, J. Membrane Sci., 22 (1985) 117-135.

11 S. Nakao, J.G. Wijmans and C.A. Smolders, Resistance to the permeate flux in unstirred ultrafiltration of dissolved macromolecular solutions, J. Membrane Sci., 26 (1986) 165-178.

12 J.P. Johnston and A.G. Ogston, A. boundary anomaly found in the ultracentrifugal sedimentation of mixtures, Trans. Faraday Soc., 42 (1946) 789-799.

13 C. Tanford and M.L. Wagner, Hydrogen ion equilibria of lysozyme, J. Amer. Chem. Soc., 76 (1954) 3331-3336.

14 J.R. Colvin, The size and shape of lysozyme, Can. J. Chem., 30 (1953) 831-834.

15 K.E. Hopper and H.A. McKenzie, Minor components of bovine $\alpha$-lactalbumin A and B, Biochim. Biophys. Acta, 295 (1973) 352-363.

16 G. Scatchard, I.H. Scheinberg and S.H. Amstrong, Physical chemistry of protein solutions. IV. The combination of human serum albumin with chloride ion, J. Amer. Chem. Soc., 72 (1950) 535-540.

17 H. Klostergaard and R.A. Pasternak, Electrophoresis and ultracentrifuge studies of milk pro teins. II. $\alpha$-Lactalbumin, J. Amer. Chem. Soc., 79 (1957) 5674-5676.

18 V.L. Vilker, C.K. Colton and K.A. Smith, The osmotic pressure of concentrated protein solutions: effect of concentration and $\mathrm{pH}$ in saline solutions of bovine serum albumin, J. Colloid Interface Sci., 79 (1981) 548-566.

19 F.M. Robbins, R.E. Andreotti, L.G. Holmes and M.J. Kronman, Inter- and intramolecular interactions of $\alpha$-lactalbumin. VII. The hydrogen ion titration curve of $\alpha$-lactalbumin, Biochim. Biophys. Acta, 133 (1967) 33-45.

20 C. Tanford, S.A. Swanson and W.S. Shore, Hydrogen ion equilibria of bovine serum albumin, J. Amer. Chem. Soc., 77 (1955) 6414-6421.

21 Y. Looze, E.D. Maes, A.O. Barel and J. Leonis, Hydrodynamic studies of some lysozymes and $\alpha$-lactalbumins, Protides Biol. Fluids, 19 (1971; publ. 1972) 409-411.

22 W.C. Gordon and W.F. Semmett, Isolation of crystalline $\alpha$-lactalbumin from milk, J. Amer. Chem. Soc., 75 (1957) 328-330.

23 R.C. Deonier and J.W. Williams, Self-association of muramidase (lysozyme) in solution at $25^{\circ}, \mathrm{pH}=7.0$ and $I=0.20$, Biochemistry, 9 (1970) $4260-4267$.

24 R.F. Steiner, Reversible association processes of globular proteins. II. Electric complexes of plasma albumin and lysozyme, Arch. Biochem. Biophys., 47 (1953) 56-75.

25 J. Rodriguez, C.H. Allibert and J.M. Chaix, A computer method for random packing of spheres of unequal size, Powder Technol., 47 (1986) 25-33.

26 R. Ben Aïm and P. Le Goff, Effet de paroi dans les empilements désordonnés de sphères et application à la porosité de mélanges binaires, Powder Technol., 1 (1967) 281-290.

27 J.A. Dodds, The porosity and contact points in multicomponent random sphere packings calculated by a simple statistical geometric model, .J. Colloid Interface Sci., 77 (1980) 317327.

28 W.M. Visscher and M. Bolsterli, Random packing of equal and unequal spheres in two and three dimensions, Nature (London), 239 (1972) 504-507. 
29 P.C. Carman, Fundamental principles of industrial filtration, Trans. Inst. Chem. Eng., 16 (1938) 168-188.

30 P. Dejmek, Concentration polarization in ultrafiltration of macromolecules, Ph.D. Thesis, Lund Institute of Technology, Lund, Sweden, 1975.

31 R.M. McDonogh, C.J.D. Fell and A.G. Fane, Surface charge and permeability in the ultrafiltration of non-flocculating colloids, J. Membrane Sci., 21 (1984) 285-294.

32 P.F. Mijnlieff and W.J.M. Jaspers, Solvent permeability of dissolved polymer material. Its direct determination from sedimentation measurements, Trans. Faraday Soc., 67 (1971) $1837-1854$.

33 H. Brauer, Grundlager der Einphasen- und Mehrphasenströmungen, Verlag Sauerländer, Aarau, Switzerland, 1971, Chap. 8.

34 J. Happel, Viscous flow in multiparticle systems; Slow motion of fluids relative to beds of spherical particles, AIChE J., 4 (1958) 197-201.

35 R. Hogg, T.W. Healy and D.W. Fuerstenau, Mutual coagulation of colloidal dispersions, Trans. Faraday Soc., 62 (1966) 1638-1651.

36 E. Barouch, E. Matijevic, T.A. King and J.M. Finlan, Heterocoagulation. II. Interaction energy of two unequal spheres, J. Colloid Interface Sci., 67 (1978) 1-9 and 70 (1979) 400.

37 D.Y.C. Chan and L.R. White, The electrostatic interaction between spherical colloidal particles - a comment on the paper by Barouch et al., J. Colloid Interface Sci., 74 (1980) 303305.

38 B.K.C. Chan and D.Y.C. Chan, Electrical double-layer interaction between spherical colloidal particles: an exact solution, J. Colloid Interface Sci., 92 (1983) 281-293.

\section{Appendix 1}

The porosity and specific resistance as a function of $\mathrm{x}_{1}$

The experimental data (the porosity $\epsilon$ as a function of the volume fraction $\phi_{1}$ of small glass particles), as obtained from data points in a plot by Ben Aïm and Le Goff [26], are given in Table A.1. These data were used to estimate the specific resistance $r$ of a concentrated layer of BSA (largest protein, $r_{\mathrm{s}}=3.64$ $\mathrm{nm}$ ) and $\alpha$-lactalbumin or lysozyme (smallest proteins, $r_{\mathrm{s}}=2.02 \mathrm{~nm}$ ). These results can also be found in the table, and it will be explained in this appendix

\section{TABLE A.1}

Experimental and calculated characteristics of a binary mixture of $\alpha$-lactalbumin $\left(r_{\mathrm{s}}=2.02 \mathrm{~nm}\right)$ and BSA $\left(r_{\mathrm{s}}=3.64 \mathrm{~nm}\right)$; for explanations see text

\begin{tabular}{|c|c|c|c|c|}
\hline \multicolumn{2}{|c|}{$\begin{array}{l}\text { From Ben Aïm [26] } \\
\text { (experimental) }\end{array}$} & \multicolumn{3}{|c|}{ Calculated } \\
\hline$\phi_{1}$ & $\epsilon$ & $x_{1}$ & $r_{\mathrm{KC}}\left(10^{19} \mathrm{~m}^{-2}\right)$ & $r_{\mathrm{H}}\left(10^{19} \mathrm{~m}^{-2}\right)$ \\
\hline 0 & 0.360 & 0 & 3.05 & 2.78 \\
\hline 0.06 & 0.339 & 0.24 & 4.27 & 4.06 \\
\hline 0.14 & 0.316 & 0.45 & 6.36 & 6.36 \\
\hline 0.25 & 0.310 & 0.62 & 8.00 & 8.11 \\
\hline 0.50 & 0.326 & 0.83 & 8.91 & 8.71 \\
\hline 1 & 0.360 & 1 & 9.88 & 9.00 \\
\hline
\end{tabular}


how these results were obtained. For reasons of convenience, in this appendix the layer will be assumed to consist of BSA and $\alpha$-lactalbumin (the same results, only about $1 \%$ difference, will be obtained for a layer with BSA and lysozyme when only the dimensions of the particles are taken into account, and not the charge).

Assuming no mutual interaction (attraction or repulsion), neglecting effects from the applied pressure and assuming the proteins to be spheres with radii equal to the Stokes radius, the specific resistance and the porosity will be calculated for such a layer of unequal-sized particles as a function of the molar fraction of small particles $x_{1}$.

The molar fraction $x_{1}$ is used as the main variable in this appendix because this is the most convenient parameter to use when the experimental flux decline indices $r_{\mathrm{bl}} / C_{\mathrm{bl}}$ have to be represented. For the mixture $\alpha$-albumin/BSA, the $x_{1}$ value can be calculated from the $\phi_{1}$ value, since the specific volumes or densities are equal, using

$\phi_{1}=V_{1} /\left(V_{1}+V_{2}\right)=m_{1} /\left(m_{1}+m_{2}\right)$

where $V_{1}$ and $V_{2}$ are the volumes occupied in the layer by the small and large particles respectively, and $m_{1}$ and $m_{2}$ are the masses of these particles. The molar fraction $x_{1}$ can now be described by

$x_{1}=\left[m_{1} / 14000\right] /\left\{\left[m_{1} / 14000\right]+\left[m_{2} / 69,000\right]\right\}=m_{1} /\left[m_{1}+0.203 m_{2}\right]$

The specific resistance can easily be calculated using the general Kozeny-Carman equation:

$r_{\mathrm{KC}}=\left[5\left(S_{0}\right)^{2}\right] \cdot\left[(1-\epsilon)^{2} / \epsilon^{3}\right]$

now calculating the specific area $S_{0}$ as [33]:

$S_{0}=3\left(\phi_{1} / r_{\mathrm{s}, 1}+\phi_{2} / r_{\mathrm{s}, 2}\right)=3\left[\phi_{1} / r_{\mathrm{s}, 1}+\left(1-\phi_{1}\right) / r_{\mathrm{s}, 2}\right]$

'The results of the calculations are given in Table A.1, while the specific resistance and the porosity as a function of $x_{1}$ are plotted in Fig. 1 (see theoretical section). The almost linear dependence of the specific resistance on $x_{1}$ is quite remarkable.

A more fundamental way to calculate the specific resistance is by the (theoretical) Happel's resistance law, which incorporates a stagnant layer around the particles [34]:

$r_{\mathrm{H}}=\left[18 \varphi_{\mathrm{e}} /\left(d_{\mathrm{p}}\right)^{2}\right] \cdot\left[(1-\epsilon)^{2} / \epsilon^{3}\right]$

where $\varphi_{\mathrm{e}}$ is a rather complicated function of the porosity and the particle diameter. This term can be described accurately, for $0 \leqslant \epsilon \leqslant 0.6$, by the empirical relation [33]:

$\varphi_{\mathrm{e}}=9.0+\left[\epsilon^{3} /(1-\epsilon)^{2}\right]$ 
When the resistance of a mixture with varying porosity has to be calculated, an additional (empirical) term $\left(\epsilon / \epsilon_{\mathrm{m}}\right)^{0.75}$ has to be added [33], where $\epsilon_{\mathrm{m}}$ is the porosity of the mixture and $\epsilon$ the original porosity.

The total resistance of a mixture can now be described by

$r_{\mathrm{H}}=\left[18 /\left(d_{\mathrm{m}}\right)^{2}\right] \cdot\left[\left(1-\epsilon_{\mathrm{m}}\right)^{2} / \epsilon_{\mathrm{m}}^{3}\right] \cdot\left\{9.0+\left[\epsilon_{\mathrm{m}}^{3} /\left(1-\epsilon_{\mathrm{m}}\right)^{2}\right]\right\} \cdot\left(\epsilon / \epsilon_{\mathrm{m}}\right)^{0.75}$

where $d_{\mathrm{m}}$ is calculated as

$d_{\mathrm{m}}=1 /\left[\phi_{1} / d_{1}+\left(1-\phi_{1} / d_{2}\right]\right.$

The calculated values of $r_{\mathrm{H}}$ as a function of $x_{1}$ are also given in Table A.1. In Fig. A.1 a comparison is given between the data on the specific resistance according to the two models. Apparently the two models result in about the same specific resistance in the porosity range used. Large deviations can be expected only when $\epsilon_{\mathrm{m}}>0.6$, i.e., when $\varphi_{\mathrm{e}}$ becomes much larger than the value represented by eqn. (A5). For reasons of convenience the simpler Kozeny-Carman model will be used to compare "theoretical" and experimental data.

Although the calculations show an almost linear dependence of the specific resistance on the molar fraction, this might be difficult to understand because of the declining average particle diameter of the mixture $d_{\mathrm{m}}$ and the decrease in porosity as well. To show the effect of only a decrease in the $d_{\mathrm{m}}$ value, in Fig. A.2 the specific resistance is plotted as a function of the molar fraction $x_{1}$ for a constant $\epsilon$ value of 0.36 and for the "actual" variation in porosity, using the experimental values of Ben Aïm [26]. (The calculations are done using the Kozeny-Carman equation.) From the figure it can be concluded that the specific resistance indeed decreases, compared to a linear dependence, when only the average particle diameter $d_{\mathrm{m}}$ decreases (lower set of data points).

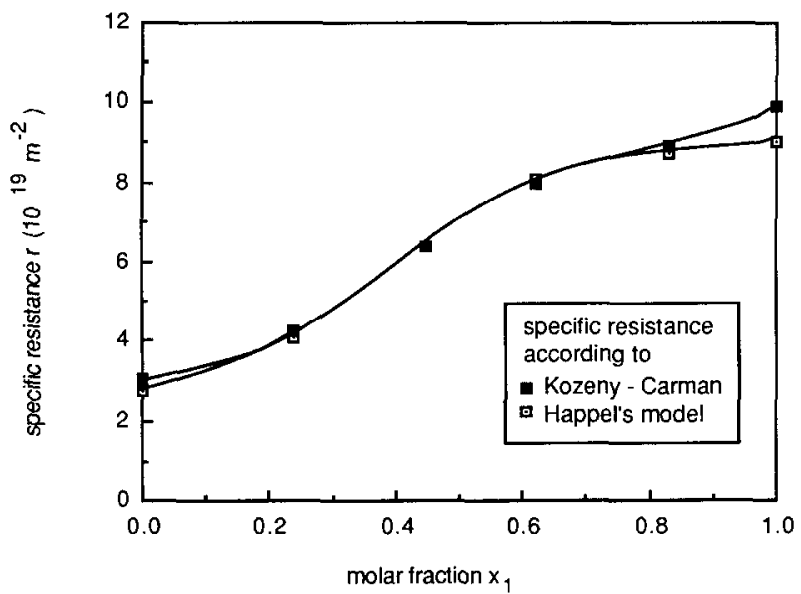

Fig. A.1. The specific resistance of a mixture of BSA and $\alpha$-lactalbumin as a function of the molar fraction of $\alpha$-lactalbumin according to two models: the Kozeny-Carman model and Happel's model. 


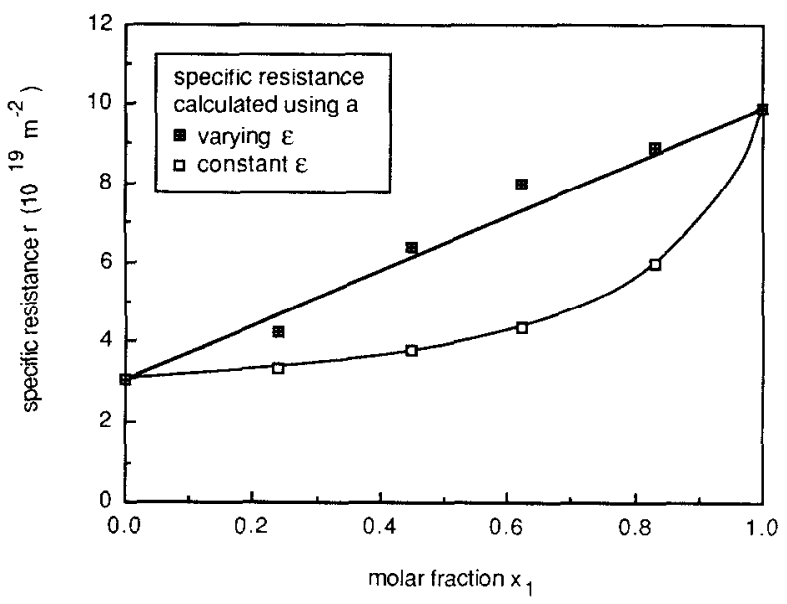

Fig. A.2. The specific resistance as a function of the molar fraction of small particles, using a varying porosity and a constant porosity.

\section{Appendix 2}

Estimation of the interparticle distance in single protein solutions and in mixtures

In this appendix the change in packing density, as a result of the charge interactions, will be estimated using the experimental ultrafiltration results. As shown in Fig. 6 the flux decline index of a mixture of lysozyme and $\alpha$ lactalbumin can be substantially larger than the flux decline index of the single protein solutions of lysozyme or $\alpha$-lactalbumin. It is therefore very likely that the porosities and the interparticle distances will also be different. They are calculated as follows: using eqn. (7) the sedimentation coefficient of the solutions can be calculated from the flux decline index by assuming the sedimentation coefficient of the mixture is equal to the sedimentation coefficient of a single protein with the same (total) concentration. From the value of the sedimentation coefficient, the concentration in the boundary layer can be calculated [eqn. (11)], and so the specific resistance $r_{\mathrm{bl}}$. By using the KozenyCarman equation [eqn. (9)] and taking $r_{\mathrm{KC}}=r_{\mathrm{b}}$, the apparent porosity $\epsilon_{\mathrm{app}}$ can be calculated. Now the minimum distance between the proteins can be estimated, using the representation of Fig. A.3 for two particles in a protein layer.

Knowing that the size of $\alpha$-lactalbumin is identical to that of lysozyme, a fairly simple model can be derived for the total porosity: assume the layer to consist of randomly packed spheres, in which the particles are present plus an extra voidage which prevents the particles from touching (caused by intercharge interactions, hydration shells or mixed forms). If the particles have a radius $r_{1}$ and the spheres have a radius $r_{2}$, the minimum interparticle distance 


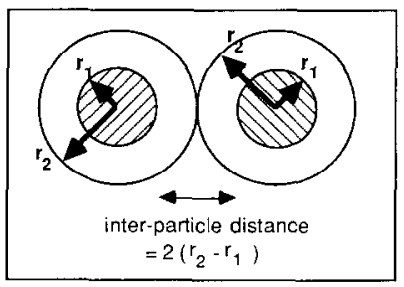

Fig. A.3. Representation of the radii and the interparticle distance of two particles. For details see text.

will be $2\left(r_{2}-r_{1}\right)$. The porosity of this system can be described as resembling the porosity of a randomly packed layer of equal spheres $(\epsilon=0.36)$ plus the extra voidage inside the spheres, $0.64\left(1-V_{1} / V_{2}\right)$, where $V_{1}$ and $V_{2}$ are the volumes of the particles and the spheres, respectively. This results in:

$\epsilon_{\text {tot }}=0.36+0.64\left[1-V_{1} / V_{2}\right]=0.36+0.64\left[1-\left(r_{1}\right)^{3} /\left(r_{2}\right)^{3}\right]$

Taking $\epsilon_{\mathrm{app}}=\epsilon_{\mathrm{tot}}$, the radius $r_{2}$ can be calculated as:

$r_{2}=r_{1}\left[0.64 /\left(1-\epsilon_{\text {tot }}\right)\right]^{\frac{1}{3}}$

from which the interparticle distance $\left[=2\left(r_{2}-r_{1}\right)\right]$ can be calculated.

For $A P=400 \mathrm{kPa}$, the experimental values of the flux decline index are $1.5 \times 10^{17} \mathrm{~m} / \mathrm{mol}$ for single protein solutions of $\alpha$-lactalbumin or lysozyme and $3.5 \times 10^{17} \mathrm{~m} / \mathrm{mol}$ (maximum) for mixtures of $\alpha$-lactalbumin and lysozyme. Using these values and eqn. (7), the sedimentation coefficients will be $2.52 \times 10^{-14}$ and $1.08 \times 10^{-14} \mathrm{sec}$, respectively, from which the concentration in the boundary layer can be calculated, via eqn. (11), as being 450 and 625 $\mathrm{kg} / \mathrm{m}^{3}$. The resulting specific resistances $r_{\mathrm{bl}}$ will then be $4.82 \times 10^{18}$ and $1.56 \times 10^{19} \mathrm{~m}^{-2}$. With $r_{\mathrm{bl}}=r_{\mathrm{KC}}$ the porosity $\epsilon_{\mathrm{app}}$ can be calculated to be 0.655 and 0.537 for the single protein solutions and the mixture, respectively.

Using eqn. (A9) the radius $r_{2}$ can be calculated; these values are $1.23 r_{1}$ and $1.11 r_{1}$, respectively. With $r_{1}=2.0 \mathrm{~nm}$ the interparticle distance will be $0.92 \mathrm{~nm}$ for the single protein solutions and $0.44 \mathrm{~nm}$ for the mixture. The latter value is the minimum value for the mixture; when other molar fractions are used the distance will be larger. When the applied pressure is $100 \mathrm{kPa}$ the distances can be calculated to be $2.48 \mathrm{~nm}$ for the single protein solutions and $1.36 \mathrm{~nm}$ as a minimum distance in a mixture of positive and negative proteins.

At the moment the interparticle distance is the subject of further research. Based on theories on the electrostatic interactions of particles [35-38], eventually followed by coagulation, the distance will be estimated. Some problems can be expected, however.

(1) Only a limited number of the required parameters used to calculate the potential functions is readily available; (2) the particles are very small, so that the boundary conditions normally used in these theories will not be satisfied, 
and (3) the fact that the particles are not ideal spherical particles, but proteins, will make the application of these theories more complicated: most proteins are ellipsoidal, and the net charge of the proteins will be a result of partly positive and partly negative domains on the protein-particle surface. 